\title{
Jasmonic Acid-Induced Changes in Brassica oleracea Affect Oviposition Preference of Two Specialist Herbivores
}

\author{
Maaike Bruinsma • Nicole M. Van Dam • \\ Joop J. A. Van Loon • Marcel Dicke
}

Received: 6 September 2006 / Accepted: 18 December $2006 /$

Published online: 2 March 2007

(C) Springer Science + Business Media, LLC 2007

\begin{abstract}
Jasmonic acid (JA) is a key hormone involved in plant defense responses. The effect of JA treatment of cabbage plants on their acceptability for oviposition by two species of cabbage white butterflies, Pieris rapae and P. brassicae, was investigated. Both butterfly species laid fewer eggs on leaves of JA-treated plants compared to control plants. We show that this is due to processes in the plant after JA treatment rather than an effect of JA itself. The oviposition preference for control plants is adaptive, as development time from larval hatch until pupation of $P$. rapae caterpillars was longer on JA-treated plants. Total glucosinolate content in leaf surface extracts was similar for control and treated plants; however, two of the five glucosinolates were present in lower amounts in leaf surface extracts of JA-treated plants. When the butterflies were offered a choice between the purified glucosinolate fraction isolated from leaf surface extracts of JA-treated plants and that from control plants, they did not discriminate. Changes in leaf surface glucosinolate profile, therefore, do not seem to explain the change in oviposition preference of the butterflies after JA treatment, suggesting that as yet unknown infochemicals are involved.
\end{abstract}

Keywords Host plant selection - Glucosinolates · Pieris rapae Pieris brassicae C Cabbage · Performance $\cdot$ Octadecanoid pathway

\section{Introduction}

Plants can be attacked by many herbivorous insects and have evolved a variety of defense strategies, including morphological barriers, synthesis of toxic or repellent secondary metabolites, and the release of synomones that attract natural enemies of the herbivores.

M. Bruinsma $(\bowtie) \cdot$ J. J. A. Van Loon · M. Dicke

Laboratory of Entomology, Wageningen University, P.O. Box 8031, 6700 EH Wageningen, The Netherlands

e-mail: maaike.bruinsma@wur.nl

N. M. Van Dam

Centre for Terrestrial Ecology, Netherlands Institute of Ecology (NIOO-KNAW), P.O. Box 40, 6666 ZG Heteren, The Netherlands 
These defenses can be constitutive, i.e., expressed independent of the presence of an attacker, or inducible, in which case defense compounds accumulate in response to attack (Karban and Baldwin, 1997). Herbivores can detect induced defensive compounds and respond by avoiding these plants, which signal lower suitability as a host plant (Landolt, 1993; De Moraes et al., 2001; Kessler and Baldwin, 2001; Meiners et al., 2005). Induced plant defense can affect herbivorous insects directly through the production of toxic compounds or indirectly through the production of cues that indicate intra- or interspecific competition for the herbivores (Schoonhoven et al., 2005). Moreover, induced plant defense signals can reduce the enemy-free space for the herbivores. For parasitoids and predators, induced infochemicals may indicate the presence of their host or prey on the plant (Turlings et al., 1990; Dicke and Vet, 1999). Phenotypic changes in individual plants may therefore affect insects at different trophic levels, and thus, the composition of the insect community and food web associated with the plant (Price et al., 1980; Van Zandt and Agrawal, 2004; Takabayashi et al., 2006).

Already in the 19th century, Kirby and Spence (1863) observed that Pieris brassicae females preferred to lay their eggs on plants devoid of eggs. Later, this was confirmed under more controlled conditions by Rothschild and Schoonhoven (1977) for both $P$. brassicae and Pieris rapae. This avoidance of infested plants is caused by a physiological response of the plant to oviposition, rather than by compounds excreted by the butterflies themselves (Blaakmeer et al., 1994). The butterflies also avoid egg deposition on leaves with feeding larvae (Rothschild and Schoonhoven, 1977). It was postulated that butterflies avoid laying eggs on herbivore-infested plants because herbivore attack induces defense compounds in plants that can influence the performance of their offspring and to reduce the risk of inter- or intraspecific competition and parasitism (Thompson and Pellmyr, 1991; Shiojiri et al., 2002). Egg-induced chemical changes in Brassica plants are also known to arrest Trichogramma parasitoids that parasitize Pieris eggs (Fatouros et al., 2005).

Oviposition-site selection involves an important behavioral decision in the life cycle of an herbivorous insect because hatching larvae have limited dispersal capacity (Renwick and Chew, 1994). $P$. rapae is a solitary butterfly that lays one egg at a time, whereas $P$. brassicae is gregarious and lays batches of about 20-100 eggs. P. rapae appears to spread the risk of larval mortality, laying few eggs within any patch. This has the advantage of being able to exploit isolated plants (Davies and Gilbert, 1985). P. brassicae, however, needs patches of plants because one large egg batch will require more than one plant for all caterpillars to develop into adults. Oviposition-site selection is performed in consecutive phases of searching and contact evaluation. P. rapae butterflies use visual, olfactory and tactile cues during these phases (Rothschild and Schoonhoven, 1977; Renwick and Radke, 1988). Acceptance of a site may be determined by the balance of positive and negative factors (Renwick and Radke, 1988). Renwick and Radke (1988) suggest that olfaction does not play a role in attraction to a host plant, but may be involved in avoidance of non-host plants. $P$. rapae and $P$. brassicae are crucifer specialists and are known to use glucosinolates, toxic secondary metabolites characteristic for Brassicaceae, as oviposition stimulants. Glucobrassicin and sinigrin are effective oviposition stimulants for P. brassicae and P. rapae (Renwick et al., 1992; Van Loon et al., 1992a).

A major signal-transduction pathway involved in induced plant defense is the octadecanoid pathway (Arimura et al., 2005). A central compound in the pathway is jasmonic acid (JA), which has an important role in direct and indirect defense in many plant species. In response to JA or methyl jasmonate (MeJA) treatment, increased concentrations of several compounds have been documented in a range of plant species, e.g., proteinase inhibitors (Moura and Ryan, 2001), polyphenol oxidases (Thaler et al., 1996), nicotine 
(Baldwin et al., 1996), trypsin inhibitors (Cipollini and Sipe, 2001), glucosinolates (Cipollini and Sipe, 2001; Van Dam et al., 2004; Mewis et al., 2005), and increased volatile emission (Boland et al., 1995; Dicke et al., 1999; Koch et al., 1999).

Herbivores are reported to be affected by JA treatment of plants. Several studies have focused on the larval stage of the herbivores and have shown reduced relative growth rates and leaf consumption (Van Dam et al., 2000; Gols et al., 2003; Van Dam et al., 2004). In field experiments, spraying of JA decreased the abundance of caterpillars, flea beetles, aphids, and thrips (Thaler et al., 2001). Other studies addressed the influence of JA application to plants on oviposition-site selection behavior of adult herbivores. These studies showed that JA application can result in induced resistance as well as induced susceptibility (Stanjek et al., 1997; Kessler and Baldwin, 2001; Lu et al., 2004).

Here, we studied how JA application affects oviposition of two specialist herbivores on cabbage, Pieris rapae L. and P. brassicae L. (Lepidoptera: Pieridae) that are closely related, yet differ drastically in the amount of eggs they put on one plant. Our study is the first to compare closely related herbivores with a different oviposition strategy, which might affect the consequences of JA-induced responses. JA is known to mediate the induction of chemical defense responses in plants to feeding damage and deposition of eggs (Dicke and Van Poecke, 2002; Hilker and Meiners, 2006; Mumm and Hilker, 2006). By using JA, we were able to examine the effects of induced defense responses in cabbage. Moreover, JA application has the advantage that visually detectable damage and the presence of herbivores or eggs are avoided. Finally, JA allows control over the strength of induction through controlled dosages. We hypothesized that JA treatment would inhibit the oviposition of the butterflies. We made solvent extracts to address the identity of the active plant compounds that influenced butterfly behavior. Rather than testing whole-leaf extracts, however, we extracted the glucosinolates from the surface of both control and JA-treated plants and tested the oviposition preference of the butterflies for these glucosinolate fractions on a neutral substrate. Furthermore, we included a control experiment to exclude a potential direct effect of JA on oviposition behavior. We addressed the following questions: (1) does JA treatment of cabbage plants affect host plant selection of the two Pieris butterfly species; (2) are there differences between solitary and gregarious butterflies; (3) does JA treatment affect glucosinolate levels in leaf surface extracts; and (4) do changes in glucosinolate levels determine the changes in oviposition preference?

\section{Methods and Materials}

Plants and Insects Brussels sprouts plants, Brassica oleracea var. gemmifera L. (Brassicaceae) cultivar Cyrus were grown from seed in a greenhouse in plastic pots $(11 \times 11 \times 11 \mathrm{~cm})$ at $20-28^{\circ} \mathrm{C}, 40-80 \% \mathrm{RH}$ and a $16-\mathrm{hr}$ light $/ 8-\mathrm{hr}$ dark photoperiod. All experiments were conducted with 6- to 7-wk-old plants. Stock colonies of the large cabbage white $P$. brassicae and the small cabbage white $P$. rapae were maintained on Brussels sprouts in a climatized room at $20-22^{\circ} \mathrm{C}, 50-70 \% \mathrm{RH}$ and a $16-\mathrm{hr}$ light/8-hr dark photoperiod.

Chemical Analysis Brussels sprouts plants were sprayed with $0.1 \mathrm{mM}$ JA or control solution. JA $(( \pm)$ jasmonic acid, purity $>97 \%$; Sigma-Aldrich, St Louis, MO, USA) was applied to the surface of the leaves, i.e., plants were sprayed with a JA solution with $0.1 \%$ Tween 20 until run-off or just with $0.1 \%$ Tween 20 for the control. The next day, glucosinolates (GLS) were extracted from the surface of the intact Brussels sprouts leaves. Each sample consisted of four leaves (between the third to sixth leaf from the base of a 
plant) that were cut at the base of the petiole. Directly after cutting, the lamina was dipped for $5 \mathrm{sec}$ in $300 \mathrm{ml}$ of dichloromethane, and after a 5-sec interval, it was dipped for $5 \mathrm{sec}$ in $150 \mathrm{ml}$ of methanol (Städler and Roessingh, 1990; Van Loon et al., 1992a; Griffiths et al., 2001). The methanol was evaporated from the crude methanol dip-volume with a rotary evaporator (IKA-Werke GmbH, Staufen, Germany). For each treatment, 11 plants were sampled. The extract was redissolved in methanol, desulphatased on a DEAE-Sephadex A25 column, and separated on a reverse phase C-18 column by using high performance liquid chromatography (HPLC) as described in Van Dam et al. (2004). Glucosinolate detection was performed with a photodiode array (PDA) detector $(200-350 \mathrm{~nm})$ with $229 \mathrm{~nm}$ as the integration wavelength. Sinigrin (sinigrin monohydrate, ACROS, NJ, USA) was used as an external standard. We used the correction factors at $229 \mathrm{~nm}$ from Buchner (1987) and the EC (EC, 1990) to calculate the concentrations of the glucosinolates. Desulfoglucosinolate peaks were identified by comparison of HPLC retention times and UV spectra with standards kindly provided by M. Reichelt, MPI Chemical Ecology and a certified rape seed standard (Community Bureau of Reference, Brussels, code BCR-367R). The surface area was measured directly after dipping, and the dry mass of the leaves was measured after drying at $50^{\circ} \mathrm{C}$ for $72 \mathrm{hr}$. The GLS content was calculated in pmol per $\mathrm{cm}^{2}$ leaf material.

Herbivore Oviposition Preference Test Pieris adults emerged from pupae in a large oviposition cage $(67 \times 100 \times 75 \mathrm{~cm})$ in a greenhouse compartment at $22-24^{\circ} \mathrm{C}$ and $50-70 \%$ RH. Apart from natural daylight, cages were illuminated by sodium vapor lamps (type SON-T, $500 \mathrm{~W}$, Philips, The Netherlands) from 8:00 A.M. until 2:00 P.M. In this cage, they were provided with a $10 \%$ sucrose solution and an oviposition substrate; depending on the experiment, a plant or an artificial leaf made of green cardboard paper sprayed with sinigrin. For the experiments, one male and one female butterfly were introduced per oviposition cage $(67 \times 50 \times 75 \mathrm{~cm})$ in the same greenhouse compartment, on the day before the experiment. In these cages, the butterflies were also provided with sucrose solution. At 8:30 A.M., the treated leaves or papers and respective controls were introduced into the cages, and the butterflies were allowed to oviposit until the beginning of the afternoon. At 2:00 P.M., the leaves were removed, and the number of eggs was counted. The experiments were carried out in several cages per day and 3-4 d per treatment with new pairs of butterflies each day, adding up to a total of 24-36 independent replicates.

Surface Application of JA The effect of JA-induced changes in Brussels sprouts plants on butterfly behavior was tested in oviposition experiments with $P$. brassicae and $P$. rapae. Three concentrations of JA solution, $0.01,0.1$, and $1 \mathrm{mM}$, corresponding to approximately $1.25,12.5$, and $125 \mu \mathrm{g} \mathrm{JA} / \mathrm{g}$ fresh weight (or $0.25,2.5$, and $25 \mathrm{nmol} \mathrm{JA} / \mathrm{cm}^{2}$ ) respectively, were sprayed on the plants and tested against a control (plants treated with $0.1 \%$ Tween 20 ). The next morning, just before the start of the experiment, the fourth, fifth, and sixth leaves from the base of the plants were cut, and their petioles were placed directly into a vial with tap water and introduced into the cages with butterflies.

Systemic Uptake of JA For P. rapae, two application methods were used to assess the effect of JA-induced changes in Brussels sprouts on oviposition preference. For the second application method, the fourth, fifth, and sixth leaves were cut from untreated plants and placed in a $0.1-\mathrm{mM}$ aqueous JA solution $22 \mathrm{hr}$ before the start of the experiment. Total uptake of the solution was on average $6.3 \pm 1.5 \mathrm{ml}$ per control leaf and $6.0 \pm 1.6 \mathrm{ml}$ for JA-treated leaves (corresponding to approximately $20 \mu \mathrm{g} \mathrm{JA} / \mathrm{g}$ fresh weight or $5 \mathrm{nmol} \mathrm{JA} / \mathrm{cm}^{2}$, assuming homogeneous distribution over the leaf tissue after uptake). 
Effect of Pure JA on Oviposition Preference In the next experiment, green cardboard paper sprayed with an oviposition stimulant was used to test the effect of pure JA on the oviposition behavior of $P$. rapae on an inert substrate. Sinigrin has been shown to be a suitable oviposition stimulant for Pieris butterflies (Van Loon et al., 1992a) and was therefore used to stimulate oviposition on the artificial substrate in this experiment. The paper $(8 \times 11.5 \mathrm{~cm})$ was treated with $1 \mathrm{ml}$ of a $5-\mathrm{mM}$ sinigrin solution (Janssen Pharmaceutica, Tilburg, The Netherlands) by spraying it with a Desaga chromatographic sprayer (Heidelberg, Germany). Subsequently, after drying, papers were sprayed with either $1 \mathrm{ml}$ of a 1-mM JA solution or water (control substrates) just before the test $(210 \mu \mathrm{g}$ $\mathrm{JA} /$ carton or $11 \mathrm{nmol} \mathrm{JA} / \mathrm{cm}^{2}$ ).

Bioassays with Purified Glucosinolate (GLS) Fractions GLS were extracted from the leaf surface as described for the chemical analysis. For each treatment, control and $0.1 \mathrm{mM} \mathrm{JA}$, 60 plants were used for the extraction, of which four to five leaves per plant were dipped. Subsequently, the extracts were fractionated following the protocol of Sørensen (Sørensen, 1990). The GLS fractions were stored in the freezer until analysis. The GLS were dissolved in methanol to make two concentrations, one corresponding to the amount of GLS extracted from the material of two plants in $0.8 \mathrm{ml}$ and one concentration corresponding to the amount of GLS from one leaf in $0.8 \mathrm{ml}$. Hereafter, we will express these concentrations in gram leaf equivalents (gle). One gle corresponds to the amount of GLS extracted from $1 \mathrm{~g}$ fresh and intact leaf. With an average weight of $6 \mathrm{~g}$ per leaf, the highest concentration corresponds to 48 gle and the lower concentration to 6 gle. With a sprayer, a volume of $0.8 \mathrm{ml}$ of one of the solutions was sprayed on green paper following the same method as described above for the test of pure JA. P. rapae butterflies were offered a two-choice situation, with one paper sprayed with GLS extracted from control plants and one paper with GLS from JA-treated plants.

Performance of $P$. rapae Caterpillars The development of first instar caterpillars to pupae was observed on control and JA-treated plants. Control plants were sprayed with a $0.1 \%$ Tween 20 solution and JA-treated plants with a solution of $0.5 \mathrm{mM} \mathrm{JA}$ with $0.1 \%$ Tween 20 . Thirty newly hatched $P$. rapae caterpillars were evenly distributed over two plants per treatment, $24 \mathrm{hr}$ after treatment, and were placed in cages $(67 \times 50 \times$ $75 \mathrm{~cm}$ ) in a greenhouse compartment at $22-24^{\circ} \mathrm{C}$ and $50-70 \% \mathrm{RH}$. The plants were replaced with new plants twice a week, so that the maximum time between induction and larval feeding never exceeded $5 \mathrm{~d}$. The number of days until pupation and pupal weight were recorded.

Statistical Analyses Each individual butterfly female was subjected to a two-choice situation, in which most individuals oviposited on both control and JA-treated leaves. As the egg load differed between individuals, the number of eggs on each treatment per individual was treated as a paired sample. The oviposition data for P. rapae were normally distributed; therefore, they were analyzed with a paired $t$-test. The oviposition data for $P$. brassicae were not normally distributed, and therefore, analyzed with the nonparametric equivalent of the paired $t$-test, the Wilcoxon matched-pair signed-ranks test. The data on the developmental time of the caterpillars in the performance test were not normally distributed and were analyzed with a Mann-Whitney $U$ test for differences between the treatments. Pupal weight was normally distributed and analyzed with an analysis of variance (ANOVA). Changes in GLS content were analyzed with a Mann-Whitney $U$ test. Statistical analyses were performed with SPSS 11.0. 
Table 1 Glucosinolate content in surface extracts of Brassica oleracea leaves in $\mathrm{pmol} / \mathrm{cm}^{2}$ for control and JA-treated plants

\begin{tabular}{lllll}
\hline Compound & $\begin{array}{l}\text { Control Treatment } \\
\text { Median (range) }\end{array}$ & $\begin{array}{l}\text { JA Treatment } \\
\text { Median (range) }\end{array}$ & $Z$ & $P$ \\
\hline Glucoiberin & $10.7(6.3-21.7)$ & $0(0-6.0)$ & -2.713 & 0.007 \\
Sinigrin & $9.3(0-23.5)$ & $4.2(0-6.8)$ & -1.774 & 0.076 \\
4-Hydroxyglucobrassicin & $0.7(0-6.1)$ & $0(0-0)$ & -2.207 & 0.027 \\
Glucobrassicin & $34.9(18.4-98.2)$ & $88.6(38.6-132.5)$ & -1.479 & 0.139 \\
4-Methoxyglucobrassicin & $0.5(0-2.5)$ & $0(0-1.3)$ & -1.370 & 0.171 \\
Total amount of & $64.1(27.0-155.7)$ & $90.0(42.9-138.8)$ & -0.563 & 0.573 \\
glucosinolates & & & & \\
\hline
\end{tabular}

\footnotetext{
${ }^{a}$ Interquartile range from first to third quartile

${ }^{\mathrm{b}} N=10$

${ }^{\mathrm{c}} N=11$
}

\section{Results}

Chemical Analysis Five GLS were detected in B. oleracea leaf surface samples: glucoiberin, sinigrin, 4-hydroxyglucobrassicin, glucobrassicin, and 4-methoxyglucobrassicin (Table 1). No significant difference was detected between JA-treated and control leaves for the total amount of GLS per $\mathrm{cm}^{2}$. The amounts of glucobrassicin, the most abundant glucosinolate in these samples, 4-methoxyglucobrassicin, and sinigrin, did not significantly differ between control and JA-treated leaves. The amounts of glucoiberin and 4-hydroxyglucobrassicin collected in the leaf surface extracts were significantly lower for JA-treated leaves compared to control leaves (Table 1). The same results were obtained when calculated for the GLS content expressed as nmol per mg dry weight (not shown).
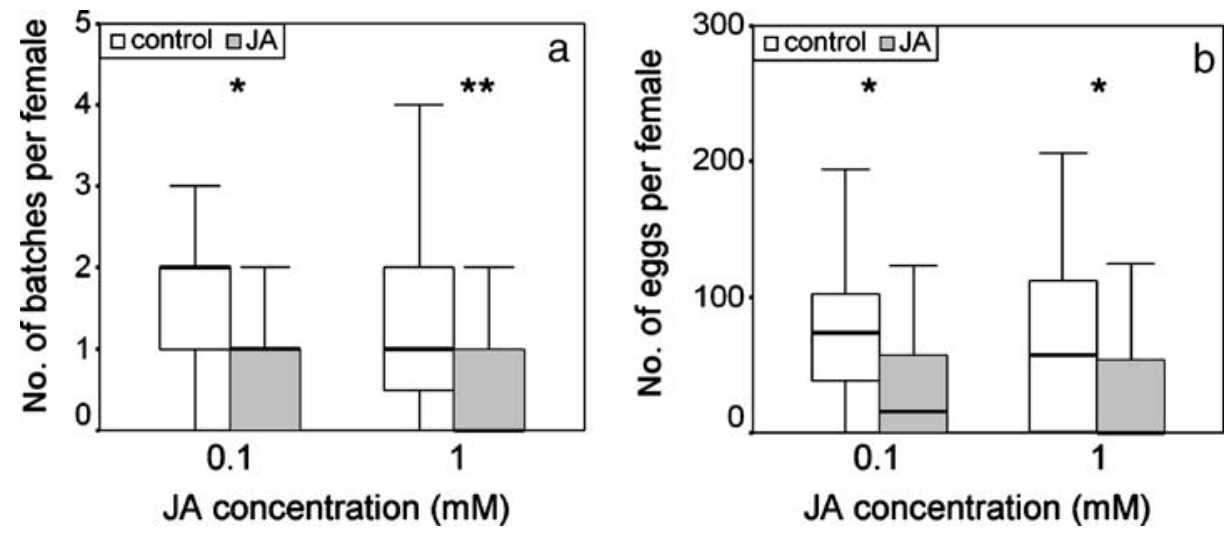

Fig. 1 Pieris brassicae oviposition on control and JA-treated plants. Two concentrations of JA were tested against a control. The box represents the interquartile range from first to third quartile, the line across the box indicates the median; asterisks indicate statistical differences between the preference for control and JAtreated plants $(* P<0.05, * * P<0.01$, Wilcoxon matched-pair signed-ranks test). a Egg batches per female per leaf. b Eggs per female per leaf 


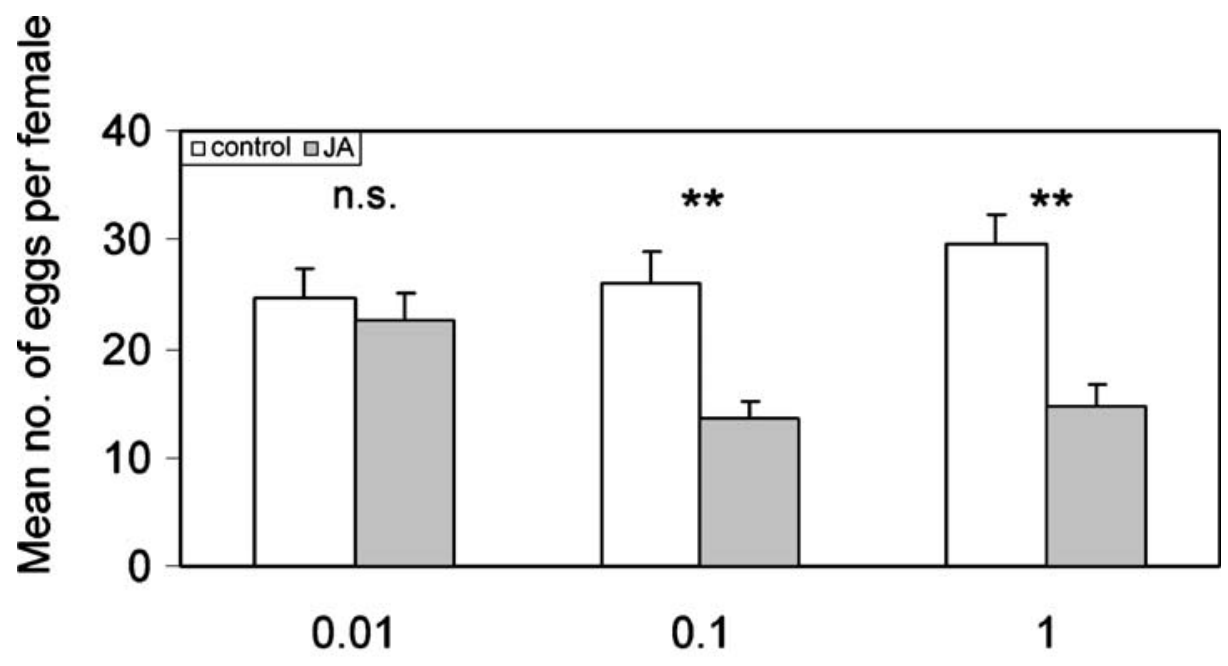

\section{JA concentration ( $\mathrm{mM})$}

Fig. 2 Pieris rapae oviposition preference (measured as the number of eggs per female per leaf) between control and JA-treated $B$. oleracea. Three concentrations of JA were tested against a control in 24 replicated experiments for each concentration. Mean numbers of eggs per female + SEM are given; asterisks indicate statistical differences between the preference for control and JA-treated plants (n.s. $P>0.05$, $* * P<0.01$, paired $t$-test)

Herbivore Oviposition Preference: Surface Application of JA As P. brassicae lays its eggs in batches, both the number of egg batches and the number of eggs per leaf were counted. For the $1 \mathrm{mM}$ JA treatment, the number of batches was significantly lower on JA-treated leaves than on control leaves $(N=36, Z=-2.628, P=0.009$, Wilcoxon matched-pair signed-ranks test), and the total number of eggs was significantly lower as well $(N=36, Z=-2.035$,

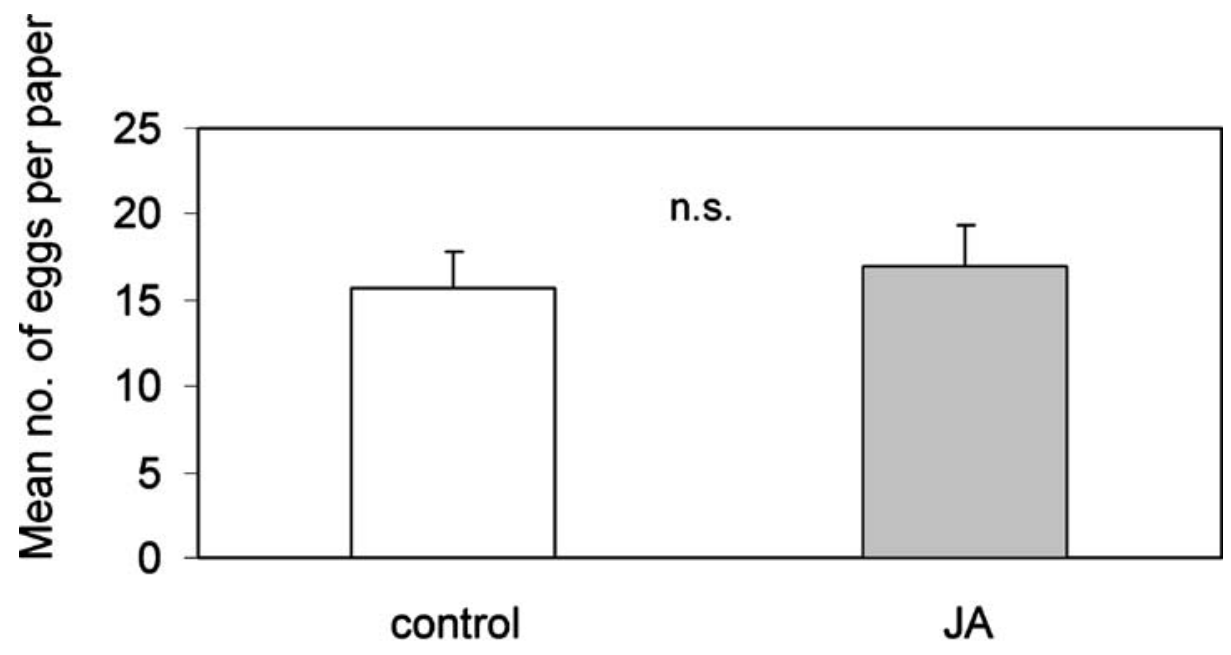

\section{Treatment}

Fig. 3 Oviposition of $P$. rapae on green paper sprayed with sinigrin plus JA and green paper with sinigrin only. Mean number of eggs per female per paper leaf + SEM (n.s. $P>0.05$, paired $t$-test, $N=27$ ) 
Table 2 Glucosinolate (GLS) content in fractions from leaf surface extracts from control and JA-treated plants in $\mathrm{pmol} / \mathrm{cm}^{2}, Z$ and $P$-values of Mann-Whitney $U$ test

\begin{tabular}{lll}
\hline Glucosinolate & GLS from control plant & GLS from JA-treated plant \\
\hline Glucoiberin & 4.9 & 3.9 \\
Sinigrin & 3.1 & 5.1 \\
4-Hydroxyglucobrassicin & Not detected & Not detected \\
Glucobrassicin & 16.0 & 81.0 \\
4-Methoxyglucobrassicin & Not detected & 0.8 \\
\hline
\end{tabular}

$P=0.042$, Wilcoxon matched-pair signed-ranks test). For the $0.1-\mathrm{mM} \mathrm{JA}$ treatment, the result was similar $(N=27$, batches: $Z=-2.223, P=0.026$; eggs: $Z=-2.138, P=0.032$, Wilcoxon matched-pair signed-ranks test) (Fig. 1). Experiments with $0.01 \mathrm{mM}$ JA application did not show discrimination by the butterflies between the treated and control leaves (results not shown). Also, P. rapae butterflies significantly preferred to oviposit on control leaves compared to JA-treated leaves (Fig. 2). The leaves treated with the two highest concentrations of JA tested, 1 and $0.1 \mathrm{mM}$, were avoided in favor of the control leaves (paired $t$-test, respectively, $t=3.805, d f=23, P=0.001$ and $t=3.681, d f=23, P=0.001$ ). The lowest concentration of JA tested, i.e., $0.01 \mathrm{mM}$, did not affect the distribution of eggs over the leaves $(t=-0.662, d f=23, P=0.52$, paired $t$-test $)$.

Systemic Uptake of JA In the experiment with P. rapae that employed systemic uptake of JA through the petiole, the same result was obtained: the number of eggs on the JA-treated leaves $(10.0 \pm 1.57)$ was lower than on the control $(19.12 \pm 2.82)$ leaves $(t=3.976, d f=31$, $P<0.001$, paired $t$-test).

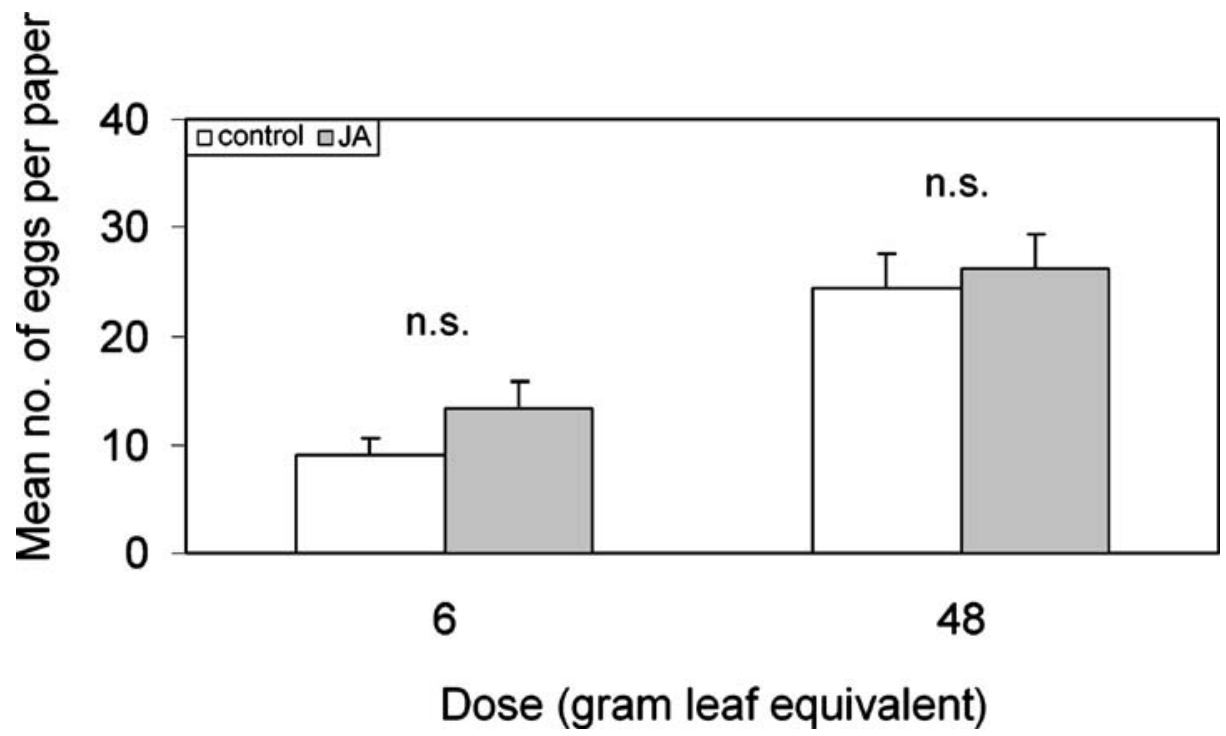

Fig. 4 Oviposition of P. rapae on green paper sprayed with purified GLS-fractions from leaf surface extracts of control and JA-treated plants. Mean number of eggs per female per paper leaf + SEM (n.s. $P>0.05$, paired $t$-test, $N=20$ ) 


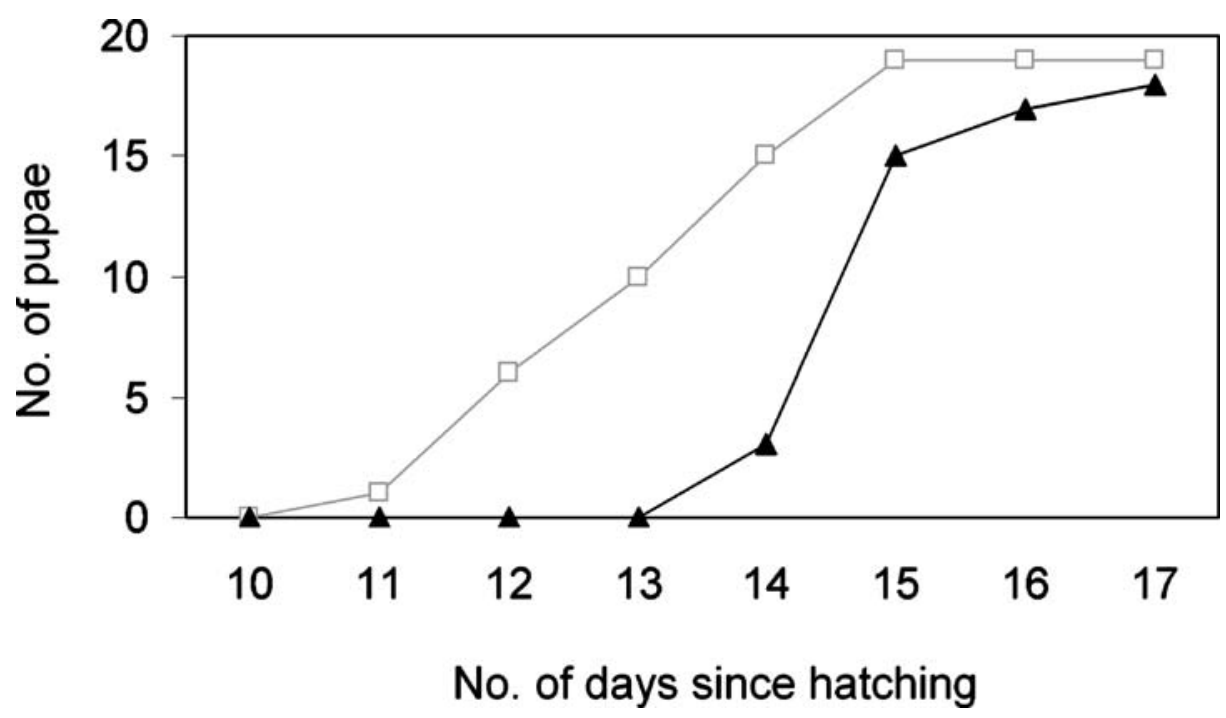

Fig. 5 Development time of $P$. rapae caterpillars from hatching until pupation on control [open squares $(\square)$ ] and JA-treated [closed triangles $(\triangleleft)$ ] plants. Cumulative number of pupae per treatment

Effect of Pure JA on Oviposition Preference When paper treated with sinigrin and JA was compared to paper with only sinigrin, there was no difference in the number of eggs the butterflies deposited on the two substrates ( $t=-0.438, d f=26, P=0.67$, paired $t$-test) (Fig. 3). These results show that the observed effect of the JA treatment on herbivore oviposition behavior was due to induced changes in leaf tissue rather than to a direct repellent or deterrent effect of JA itself.

Bioassays with Purified Glucosinolate Fractions The butterflies did not discriminate between the two GLS fractions (Table 2), the number of eggs on paper with the GLS from control plants, and the number of eggs on paper with GLS from JA-treated plants was not different for either concentration (concentration 6 gle: $Z=-1.514, N=20, P=0.130$, Wilcoxon matched-pair signed-ranks test; 48 gle: $t=-0.523, d f=19, P=0.607$, paired $t$-test) (Fig. 4).

Performance of $\mathrm{P}$. rapae Caterpillars About two-thirds of the caterpillars survived until pupation, and a similar number of caterpillars reached the pupal stage on both treatments, 19 on control and 18 on JA-treated plants (Fig. 5). The caterpillars on JA-treated plants pupated on average after $15 \mathrm{~d}$, while the caterpillars on the control plants pupated significantly sooner, on average after $13 \mathrm{~d}$ (Mann-Whitney $U, Z=-4.071, P<0.001$ ). The average pupal weight on the control plants, $165 \pm 3.4 \mathrm{mg}$, was similar to that on the JA-treated plants, $158 \pm 3.3 \mathrm{mg}$ (ANOVA, $F=2.665, d f=1, P=0.112$ ).

\section{Discussion}

Our data show that JA treatment of Brussels sprouts leaves reduces the acceptance of leaves for oviposition by $P$. rapae and $P$. brassicae in a similar way. Treatment of leaves with 0.1 or 
$1 \mathrm{mM}$ JA reduced the proportion of eggs the butterflies laid on these leaves. A concentration of $0.01 \mathrm{mM}$ JA did not change oviposition preference. The former concentrations are comparable to the concentrations of JA or MeJA that were applied to several plant species in other studies and that reduced development of Spodoptera exigua, Trichoplusia ni, Manduca sexta, thrips, and aphids (Thaler et al., 1996; Avdiushko et al., 1997; Van Dam et al., 2000; Omer et al., 2001), and abundance of Manduca quinquemaculata, S. exigua, thrips, and flea beetles in the field (Kessler and Baldwin, 2001; Thaler et al., 2001). For cabbage plants, Lu et al. (2004) found inducible resistance in a susceptible Brassica species (Chinese cabbage, Brassica campestris L.) and induced susceptibility in a resistant Brassica species (common cabbage, $B$. oleracea) for Plutella xylostella L.

To exclude that JA itself caused the above effect, we tested the phytohormone on an inert substrate and studied two different application methods to the leaf material. We considered this an essential control that is lacking in other studies. The results of these experiments show that it was not JA itself that caused the difference in oviposition preference between control and JA-treated leaves, thus providing proof that processes in the plant induced by the JA treatment changed the acceptability of the leaves. It has also been reported previously that development of cabbage looper or tobacco hornworm larvae is not affected when MeJA is added to an artificial diet, but it is retarded when MeJA was applied to cabbage or tobacco plants (Avdiushko et al., 1997).

In leaf surface extracts of JA-treated and untreated Brussels sprouts, we found five glucosinolates. After JA application, glucobrassicin, the major glucosinolate in the $B$. oleracea cultivar we used, occurred at a level twice as high as in control plants, and glucoiberin and 4-hydroxyglucobrassicin concentrations decreased. The total glucosinolate content did not change significantly. However, most other studies on glucosinolate content in Brassicaceous plants after induction by JA- or MeJA-treatment or insect attack have reported an increase in glucosinolates, although there is substantial variation among different species, or even genotypes, and type of induction (Bodnaryk, 1994; Cipollini and Sipe, 2001; Mikkelsen et al., 2003; Mewis et al., 2005). Moreover, glucosinolates may not be evenly distributed throughout the leaf. We measured glucosinolate content in a surface extract after $24 \mathrm{hr}$, whereas most studies have measured glucosinolate content in whole leaf extracts and after a longer induction time. Recently, Reifenrath et al. (2005) postulated that the wax layer does not contain glucosinolates, and the polar glucosinolates that are found by using the solvent extraction method are washed from the inner leaf to the outside through the stomata. Nevertheless, we chose a surface extraction method because the butterflies retrieve chemosensory information from the leaf surface, as they do not damage the leaf before ovipositing. Surface extracts are thus likely to give a better reflection of the chemosensory information used than whole leaf extracts.

Both butterfly species distinguish between induced and non-induced leaves, most likely based on chemical differences, as JA-induced leaves do not display herbivore presence or damage. The different levels of two out of five glucosinolates in the surface extracts may provide a chemosensory basis for the oviposition preference observed, although the isolated GLS from the two treatments yielded no differences in acceptance of the paper for oviposition. While the isolated GLS on paper stimulated oviposition behavior, they appear not to be the main cue to discriminate between the JA-induced and non-induced cabbage plants.

We did not quantify other chemicals, stimulants, deterrents, or precursors that might mediate preference behavior, such as isothiocyanates, terpenoids, other glycosides, or amino acids (Huang et al., 1993; Renwick and Chew, 1994; Soldaat et al., 1996; Agrawal and Kurashige, 2003). Both P. rapae and P. brassicae can perceive a broad range of chemicals (Van Loon et al., 1992b; Hern et al., 1996). Electroantennogram responses to a 
range of plant volatiles are similar for both species (Van Loon et al., 1992b), although host plant selection by Pieris butterflies appears largely based on contact chemoreception rather than olfaction (Renwick and Chew, 1994). Host plant selection is suggested to depend on a balance of stimulants and deterrents and not just on the detection of presence or absence of particular compounds (Huang et al., 1993; Bruce et al., 2005). Therefore, glucosinolates, in combination with other stimulants or deterrents, may determine the acceptance of a host plant by the butterflies.

The octadecanoid pathway, in which JA is a key molecule, is involved in induction of synomones in response to oviposition and to herbivore damage (Meiners and Hilker, 2000; Dicke and Van Poecke, 2002; Hilker and Meiners, 2006; Mumm and Hilker, 2006). JA treatment of plants results in emission of synomones that attract natural enemies like predatory mites and parasitoids (Dicke et al., 1999; Hilker and Meiners, 2002; Van Poecke and Dicke, 2002; Hilker and Meiners, 2006). This attraction results in a higher natural enemy density around damaged plants, and therefore, it is advantageous for the herbivores to avoid oviposition on induced plants. Moreover, intact plants lack competitors, either intra- or interspecific. Herbivores may use induced plant cues to detect the presence or absence of other herbivores, especially because plant cues are, although less reliable, often easier to detect than cues from the herbivores themselves (Vet and Dicke, 1992).

Furthermore, induced plants may affect herbivores directly by influencing the performance of their offspring. For P. rapae, the development time differed between caterpillars feeding on JA-induced and caterpillars feeding on non-induced plants. Development of the caterpillars to pupae took longer on the induced plants, which exposes them to natural enemies for a longer time and gives them a disadvantage in the competition for resources with other herbivores. These results comply with those of Agrawal and Kurashige (2003), who showed that growth of $P$. rapae larvae was reduced on herbivore-induced Brassicaceae.

In summary: (1) JA treatment of B. oleracea results in avoidance of host plants by the two Pieris butterflies; (2) the related gregarious and solitary butterfly species tested responded in a similar fashion to JA-treated plants; (3) JA treatment reduced the contents of two out of five glucosinolates in leaf surface extracts of Brussels sprouts; and (4) the purified GLS fractions could not explain the observed avoidance behavior. The results indicate that JA-induced infochemicals play an important role in host plant selection behavior of these butterflies; however, the phytochemicals involved still have to be elucidated.

Acknowledgments We thank Niels Agerbirk for advice on GLS isolation; Ciska Raaijmakers for technical assistance with the chemical analysis; Leo Koopman, Frans van Aggelen, André Gidding, and PPW for the supply of insects and plants; Erik Poelman, Tjeerd Snoeren, and Adriana Alvarez for comments and discussion; and the undergraduate students who participated in some of the experiments: Paul Egan, Eloy Erro, Olivia Garcia Gonzales, and Pascal van der Sandt. This work was supported by a VICI grant from the Netherlands Organization for Scientific Research, NWO (865.03.002).

\section{References}

Agrawal, A. A. and Kurashige, N. S. 2003. A role for isothiocyanates in plant resistance against the specialist herbivore Pieris rapae. J. Chem. Ecol. 29:1403-1415.

Arimura, G., Kost, C., and Boland, W. 2005. Herbivore-induced, indirect plant defences. Biochim. Biophys. Acta 1734:91-111.

Avdiushko, S. A., Brown, G. C., Dahlman, D. L., and Hildebrand, D. F. 1997. Methyl jasmonate exposure induces insect resistance in cabbage and tobacco. Environ. Entomol. 26:642-654.

BAldwin, I. T., Schmelz, E. A., and Zhang, Z. P. 1996. Effects of octadecanoid metabolites and inhibitors on induced nicotine accumulation in Nicotiana sylvestris. J. Chem. Ecol. 22:61-74. 
Blaakmeer, A., Hagenbeek, D., Van Beek, T. A., De Groot, A., Schoonhoven, L. M., and Van Loon, J. J. A. 1994. Plant response to eggs vs. host marking pheromone as factors inhibiting oviposition by Pieris brassicae. J. Chem. Ecol. 20:1657-1665.

BODNARYK, R. P. 1994. Potent effect of jasmonates on indole glucosinolates in oilseed rape and mustard. Phytochemistry 35:301-305.

Boland, W., Hopke, J., Donath, J., Nüske, J., and Bublitz, F. 1995. Jasmonic acid and coronatin induce odor production in plants. Angew. Chem. Int. Ed. Engl. 34:1600-1602.

Bruce, T. J. A., WAdhams, L. J., and WoodCOCK, C. M. 2005. Insect host location: a volatile situation. Trends Plant Sci. 10:269-274.

BUCHNER, R. 1987. Approach to determination of HPLC response factors for glucosinolates, pp. 50-58, in J.-P. Wathelet (eds.). Glucosinolates in Rapeseeds. Martinus Nijhoff Publishers, Dordrecht.

CIPOLlinI, D. F. and SIPE, M. L. 2001. Jasmonic acid treatment and mammalian herbivory differentially affect chemical defenses and growth of wild mustard (Brassica kaber). Chemoecology 11:137-143.

DAVIES, C. R. and GILBERT, N. 1985. A comparative study of the egg-laying behaviour and larval development of Pieris rapae L. and Pieris brassicae L. on the same host plants. Oecologia 67:278-281.

De Moraes, C. M., Mescher, M. C., and Tumlinson, J. H. 2001. Caterpillar-induced nocturnal plant volatiles repel conspecific females. Nature 410:577-580.

Dicke, M. and VAN PoEcKe, R. M. P. 2002. Signaling in plant-insect interactions: signal transduction in direct and indirect plant defence, pp. 289-316, in D. Scheel and C. Wasternack (eds.). Plant Signal Transduction. Oxford University Press, Oxford, UK.

Dicke, M. and VET, L. E. M. 1999. Plant-carnivore interactions: evolutionary and ecological consequences for plant, herbivore and carnivore, pp. 483-520, in H. Olff, V. K. Brown, and R. H. Drent (eds.). Herbivores: Between Plants and Predators. Blackwell Science, Oxford, UK.

Dicke, M., Gols, R., Ludeking, D., and Posthumus, M. A. 1999. Jasmonic acid and herbivory differentially induce carnivore-attracting plant volatiles in lima bean plants. J. Chem. Ecol. 25:1907-1922.

EC 1990. Oil seeds - determination of glucosinolates high performance liquid chromatography. OJL 170:27-34.

Fatouros, N. E., Bukovinszkine'Kiss, G., Kalkers, L. A., Gamborena, R. S., Dicke, M., and HilKer, M. 2005. Oviposition-induced plant cues: do they arrest Trichogramma wasps during host location? Entomol. Exp. Appl. 115:207-215.

Gols, R., Roosjen, M., Dijkman, H., and Dicke, M. 2003. Induction of direct and indirect plant responses by jasmonic acid, low spider mite densities or a combination of jasmonic acid treatment and spider mite infestation. J. Chem. Ecol. 29:2651-2666.

Griffiths, D. W., Deighton, N., Birch, A. N. E., Patrian, B., Baur, R., and Städler, E. 2001. Identification of glucosinolates on the leaf surface of plants from the Cruciferae and other closely related species. Phytochemistry 57:693-700.

Hern, A., Edwards-Jones, G., and MCKinlay, R. G. 1996. A review of the pre-oviposition behaviour of the small cabbage white butterfly, Pieris rapae (Lepidoptera: Pieridae). Ann. Appl. Biol. 128:349-371.

HiLKER, M. and MEINERS, T. 2002. Induction of plant responses to oviposition and feeding by herbivorous arthropods: a comparison. Entomol. Exp. Appl. 104:181-192.

Hilker, M. and Meiners, T. 2006. Early herbivore alert: insect eggs induce plant defense. J. Chem. Ecol. 32:1379-1397.

HuANG, X. P., RENWICK, J. A. A., and SAChDEV-GuPTA, K. 1993. Oviposition stimulants and deterrents regulating differential acceptance of Iberis amara by Pieris rapae and P. napi oleracea. J. Chem. Ecol. 19:1645-1663.

KARBAN, R. and BALDWIN, I. T. 1997. Induced Responses to Herbivory. The University of Chicago Press, Chicago.

Kessler, A. and BALDWIN, I. T. 2001. Defensive function of herbivore-induced plant volatile emissions in nature. Science 291:2141-2144.

Kirby, W. and Spence, W. 1863. An Introduction to Entomology. 7 edn. Longman, Green, Longman, Roberts and Green, London.

Koch, T., Krumm, T., Jung, V., Engelberth, J., and Boland, W. 1999. Differential induction of plant volatile biosynthesis in the lima bean by early and late intermediates of the octadecanoid-signaling pathway. Plant Physiol. 121:153-162.

LANDOLT, P. J. 1993. Effects of host plant leaf damage on cabbage looper moth attraction and oviposition. Entomol. Exp. Appl. 67:79-85.

LU, Y. B., LiU, S. S., LiU, Y. Q., Furlong, M. J., and ZALuCKi, M. P. 2004. Contrary effects of jasmonate treatment of two closely related plant species on attraction of and oviposition by a specialist herbivore. Ecol. Lett. 7:337-345.

MEINERS, T. and HiLKER, M. 2000. Induction of plant synomones by oviposition of a phytophagous insect. J. Chem. Ecol. 26:221-232.

Meiners, T., Hacker, N. K., Anderson, P., and Hilker, M. 2005. Response of the elm leaf beetle to host plants induced by oviposition and feeding: the infestation rate matters. Entomol. Exp. Appl. 115:171-177. 
Mewis, I., Appel, H. M., Hom, A., RAinA, R., and Schultz, J. C. 2005. Major signaling pathways modulate Arabidopsis glucosinolate accumulation and response to both phloem-feeding and chewing insects. Plant Physiol. 138:1149-1162.

Mikkelsen, M. D., Petersen, B. L., Glawischnig, E., Jensen, A. B., Andreasson, E., and Halkier, B. A. 2003. Modulation of CYP79 genes and glucosinolate profiles in Arabidopsis by defense signaling pathways. Plant Physiol. 131:298-308.

MOURA, D. S. and RYAN, C. A. 2001. Wound-inducible proteinase inhibitors in pepper. Differential regulation upon wounding, systemin, and methyl jasmonate. Plant Physiol. 126:289-298.

Mumm, R. and HiLKer, M. 2006. Direct and indirect chemical defence of pine against folivorous insects. Trends Plant Sci. 11:351-358.

Omer, A. D., Granett, J., Karban, R., and Villa, E. M. 2001. Chemically-induced resistance against multiple pests in cotton. Int. J. Pest Manag. 47:49-54.

Price, P. W., Bouton, C. E., Gross, P., McPheron, B. A., Thompson, J. N., and Weis, A. E. 1980. Interactions among three trophic levels: influence of plants on interactions between insect herbivores and natural enemies. Annu. Rev. Ecol. Syst. 11:41-65.

REIFENRATH, K., RIEDERER, M., and MÜLLER, C. 2005. Leaf surface wax layers of Brassicaceae lack feeding stimulants for Phaedon cochleariae. Entomol. Exp. Appl. 115:41-50.

RENWICK, J. A. A. and CHEW, F. S. 1994. Oviposition behavior in Lepidoptera. Annu. Rev. Entomol. 39:377-400.

RENWICK, J. A. A. and RADKE, C. D. 1988. Sensory cues in host selection for oviposition by the cabbage butterfly, Pieris rapae. J. Insect Physiol. 34:251-257.

Renwick, J. A. A., RAdKe, C. D., SAChdeV-GuptA, K., and StÄDler, E. 1992. Leaf surface chemicals stimulating oviposition by Pieris rapae (Lepidoptera: Pieridae) on cabbage. Chemoecology 3:33-38.

Rothschild, M. and Schoonhoven, L. M. 1977. Assessment of egg load by Pieris brassicae (Lepidoptera: Pieridae). Nature 266:352-355.

Schoonhoven, L. M., VAn Loon, J. J. A., and Dicke, M. 2005. Insect-Plant Biology. 2nd edn. Oxford University Press, Oxford.

SHIOJIRI, K., TAKABAYASHI, J., YANO, S., and TAKAFUJI, A. 2002. Oviposition preferences of herbivores are affected by tritrophic interaction webs. Ecol. Lett. 5:186-192.

SoldAat, L. L., Boutin, J. P., and DerRidJ, S. 1996. Species-specific composition of free amino acids on the leaf surface of four Senecio species. J. Chem. Ecol. 22:1-12.

SørEnSEN, H. 1990. Glucosinolates: structure-properties-function, pp. 149-172, in F. Shahidi (ed.). Canola and Rapeseed: Production, Chemistry, Nutrition, and Processing Technology. Van Nostrand Reinhold, New York.

STÄDLER, E. and RoESSINGH, P. 1990. Perception of surface chemicals by feeding and ovipositing insects. Symp. Biol. Hung. 39:71-86.

StanjeK, V., Herhaus, C., Ritgen, U., Boland, W., and Städler, E. 1997. Changes in the leaf surface chemistry of Apium graveolens (Apiaceae) stimulated by jasmonic acid and perceived by a specialist insect. Helv. Chim. Acta 80:1408-1420.

Takabayashi, J., Sabelis, M. W., Janssen, A., Shiojiri, K., and VAn WiJK, M. 2006. Can plants betray the presence of multiple herbivore species to predators and parasitoids? The role of learning in phytochemical information networks. Ecol. Res. 21:3-8.

Thaler, J. S., Stout, M. J., Karban, R., and Duffey, S. S. 1996. Exogenous jasmonates simulate insect wounding in tomato plants (Lycopersicon esculentum) in the laboratory and field. J. Chem. Ecol. 22:1767-1781.

Thaler, J. S., Stout, M. J., Karban, R., and Duffey, S. S. 2001. Jasmonate-mediated induced plant resistance affects a community of herbivores. Ecol. Entomol. 26:312-324.

Thompson, J. N. and Pellmyr, O. 1991. Evolution of oviposition behavior and host preference in Lepidoptera. Annu. Rev. Entomol. 36:65-89.

Turlings, T. C. J., Tumlinson, J. H., and LEWIS, W. J. 1990. Exploitation of herbivore-induced plant odors by host-seeking parasitic wasps. Science 250:1251-1253.

VAN DAM, N. M., HADWICH, K., and BALDWIN, I. T. 2000. Induced responses in Nicotiana attenuata affect behavior and growth of the specialist herbivore Manduca sexta. Oecologia 122:371-379.

VAN DAM, N. M., WiTJES, L., and SvATOŠ, A. 2004. Interactions between aboveground and belowground induction of glucosinolates in two wild Brassica species. New Phytol. 161:801-810.

Van Loon, J. J. A., BlaAkmeer, A., Griepink, F. C., Van Beek, T. A., Schoonhoven, L. M., and De Groot, A. 1992a. Leaf surface compound from Brassica oleracea (Cruciferae) induces oviposition by Pieris brassicae (Lepidoptera: Pieridae). Chemoecology 3:39-44.

VAn LoOn, J. J. A., FrentZ, W. H., and EeuwiJK, F. A. 1992b. Electroantennogram responses to plant volatiles in two species of Pieris butterflies. Entomol. Exp. Appl. 62:253-260. 
VAn Poecke, R. M. P. and Dicke, M. 2002. Induced parasitoid attraction by Arabidopsis thaliana: involvement of the octadecanoid and the salicylic acid pathway. J. Exp. Bot. 53:1793-1799.

VAN ZANDT, P. A. and Agrawal, A. A. 2004. Community-wide impacts of herbivore-induced plant responses in milkweed (Asclepias syriaca). Ecology 85:2616-2629.

VET, L. E. M. and Dicke, M. 1992. Ecology of infochemical use by natural enemies in a tritrophic context. Annu. Rev. Entomol. 37:141-172. 